\title{
INVESTIGATION OF LOCALLY ANHARMONIC MODELS OF STRUCTURAL PHASE TRANSITIONS. SEMINUMERICAL APPROACH
}

\author{
I.V.Stasyuk, K.O.Trachenko \\ Institute for Condensed Matter Physics \\ of the National Academy of Sciences of Ukraine \\ 1 Svientsitskii St., 290011 Lviv-11, Ukraine
}

Received January 24, 1997

\begin{abstract}
In the context of description of the structural phase transition in solids, the anharmonic potential effecting on the ion in the crystal lattice is selected in the form that in addition to quadric includes cubic anharmonicity, thus possessing more general non-symmetrical form and in the symmetrical form with the Gaussian barrier. The approach based on the numerical diagonalization of the one-site Hamiltonian matrix is applied. The peculiarity of the energy spectrum of the system with the Gaussian barrier potential, particularly the existence of the parts of the spectrum with the quasidoublets or quasi-equally distanced levels structure which crossover takes place in the narrow energy interval, is established. The dependencies of the order parameter, free energy and dielectric susceptibility on the externally applied field and the order parameter and free energy on the temperature are derived for various parameters of both models. The phase diagram (temperature-field) is derived and the influence of the anharmonicity on the form of the phase coexistence curve and location of the critical point is studied. It is shown that the asymmetry of the local potential results in the deviation of the phase coexistence curve from the vertical line in the plane (temperature-field) and in the appearing of the first order phase transition occurring as the temperature varies.
\end{abstract}

\section{Introduction}

While studying the mechanism of the structural phase transitions in crystals, the problem of choosing the type of the local potential $V(q)$ in the model Hamiltonian

$$
H=\sum_{i}\left(\frac{p_{i}^{2}}{2 M}+V\left(q_{i}\right)\right)-\frac{1}{2} \sum_{i j} \varphi_{i j} q_{i} q_{j},
$$

describing a certain mode of the lattice vibrations which is active at the phase transition, is raised. We use the concept of the local normal coordinate denoted here by $q_{i}$ (see, for example, [1]).

In the harmonic approximation $\left(V(q)=\frac{\alpha}{2} q^{2}, \alpha=M \omega_{0}^{2}\right)$, the crystal lattice may become unstable in the case of the negative value of $\alpha$. As it is mentioned in a number of papers, including [1], the simplest stabilizing interaction may be chosen in the form 


$$
V\left(q_{i}\right)=-\frac{|\alpha| q_{i}^{2}}{2}+\frac{\beta q_{i}^{4}}{4} .
$$

In this case the system undergoes the structural phase transition which can be of the displacive or order-disorder type, depending on the values of parameters in the initial Hamiltonian. Such a model is often applied to the description of the phase transition in ferroelectrics.

A large number of papers was dedicated to studies of the thermodynamics and dynamics of the model described by the Hamiltonian (1), (2). Along with the application of the analytical methods of various degrees of approximation, including the method of self-consistent phonons, the method of renormalized group, etc., the attempts to take into account the effects caused by the local anharmonicity of the model by using numerical methods were made. Particularly, in ref. [2] the energy spectrum of the single-ion system described by (2) was calculated, while the interaction between the ions in different cells was taken into account in the mean field approximation; the comparison with the results obtained in the self-consistent phonons approximation was made. This approach is justified in the case of long-range interaction $\varphi_{i j}$; the obtained results can be improved by including of the higher order corrections when the expansion in powers of the inverse radius of interaction is used.

In addition to the description of ion motion in ferroelectrics, the model local anharmonic concept is applied to the description of the lattice anharmonicity in the high temperature superconductors. As it is pointed out in [3], such an anharmonicity is inherent to the motion of the apex oxygen ions in $\mathrm{YBaCuO}$ and other superconductive compounds. To take into consideration such an anharmonicity in the case of local double-well potential, the approach which introduces pseudospin variables describing the vibrational degrees of freedom was used. The pseudospin-electron model derived in this way, describing also the interaction of the conducting electrons with local anharmonic mode, was the subject of intensive study in the last years [4-6]. The asymmetry of the anharmonic potential which is characteristic for the systems of this kind was taken into account by including into the Hamiltonian the term which described the interaction with some internal field $\tilde{h}_{i}$, depending on the occupancy of electronic states $\left(\tilde{h}_{i}=h+g n_{i}\right)$. Structural phase transitions in HTSC systems, including $H g$-based superconductors, have been recently of wide consideration, in the context of the reported connection between the lattice softening in these compounds and transition to the superconducting state. Particularly, in ref. [7] it was mentioned that near the transition point an anomalous abrupt mode softening was observed. The peculiarities related to the presence of the lattice anharmonicity were observed for the $\mathrm{YBaCuO}$ series on the temperature dependencies of the lattice constant $c$, coefficients of thermal expansion in the direction of anharmonic vibrations of oxygen ions, as well as specific heat, thermoconductivity and velocity of ultrasonic waves in the form of jump-like and hysteresis behaviour (see [8]).

For the description of such situations, the model potential possessing more general non-symmetrical shape due to the presence of the cubic term, was considered in [8-9]:

$$
V\left(q_{i}\right)=\frac{\alpha q_{i}^{2}}{2}-\frac{\beta q_{i}^{3}}{3}+\frac{\gamma q_{i}^{4}}{4}, \alpha>0
$$

(so called " $\varphi^{3}+\varphi^{4}$ " model ${ }^{1}$ ). Basing on (3) and applying the method of self-consistent phonons it was shown that the behaviour of the apex oxy-

\footnotetext{
${ }^{1}$ The potential of the form (3) occurs also as the effective one in the free energy functional at the description of liquid-gas transition [10], and in other similar cases.
} 
gen in $\mathrm{YBa}_{2} \mathrm{Cu}_{3} \mathrm{O}_{7-\delta}$ compound is bistable. The dependence of the order parameter on the temperature was shown to be of a hysteresis character. However, the question of the applicability of the method of self-consistent phonons to the description of the phase transition in the systems described by (3), including critical areas arises, (see [1]).

Another possibility to describe the anharmonic potential acting on the ion in the crystal lattice is to write it as a superposition of a Gaussian and a harmonic oscillator potential:

$$
V_{G}\left(q_{i}\right)=\frac{\alpha q_{i}^{2}}{2}+C e^{-B q_{i}^{2}},
$$

which has two symmetrically located minima if $\frac{\alpha}{2 B C}<1$.

This potential was applied, for example, in [11] and [12] to consider the crystal dynamics and phase transition in ferroelectrics within the selfconsistent phonon field approximation. Since the role of the anharmonic interaction in (3) is not essential if the temperature is high, or when $\left.\left.\left\langle q^{2}\right\rangle\right\rangle\right\rangle$ $(1 / \beta)$, the author of [6] identifies potential (3) as the "low-temperature anharmonicity potential".

The potential of $V_{G}\left(q_{i}\right)$ type has been used also in the theory of molecules. In [13] the numerical treatment of (4), similar to [2], was carried out; the low-lying energy levels were derived by solving the secular equations for various sets of potential parameters. The latter one were then derived for certain configurations of some molecules.

The main aim of this paper is the further development of the numerical approaches mentioned above. We model the potential acting on the ion in the form (3) and (4) and consider the anharmonic character of the ion motion by the numerical diagonalization of the Hamiltonian matrix (1). The interaction between ions in different cells we allow for in the mean-field approximation, assuming the long-range character of the intercell interaction. Basing on this approach, we derive the dependencies of the order parameter, free energy and dielectric susceptibility on the externally applied field and of the order parameter and free energy on the temperature for various model parameters. Analysing these dependencies we investigate the phase transition as the external field varies as well as the temperature; in the case of the potential in form (3) it is of the first order; we also consider the behaviour of the dielectric susceptibility near the phase transition point. Phase transition in the system described by (4) is of the first order, as the external field varies, and second order, at the temperature change. In addition, we construct the phase diagrams (external field, temperature) and study the influence of the anharmonicity of each of potentials (3), (4) on the form of the phase diagrams and the location of the critical points.

\section{2. " $\varphi^{3}+\varphi^{4} "$ Model}

\subsection{Model Hamiltonian}

In this section we consider the case of local anharmonic potential in nonsymmetric form (3), where $\alpha=M \omega_{0}^{2}>0$. Transformation of the last term in (1) in accordance with the mean-field approximation leads to

$$
-\frac{1}{2} \sum_{i j} \varphi_{i j} q_{i} q_{j} \quad \rightarrow \quad \varphi\langle q\rangle q-\frac{1}{2} \varphi\langle q\rangle^{2}
$$




$$
\varphi=-\sum_{i} \varphi_{i j}
$$

This results in the following form of $H$ :

$$
\begin{aligned}
H & =\sum_{i} H_{i} \\
H_{i} & =\frac{p_{i}^{2}}{2 M}+\frac{M \omega_{0}^{2}}{2} q_{i}^{2}-\frac{\beta q_{i}^{3}}{3}+\frac{\gamma q_{i}^{4}}{4}+d q_{i}+\varphi\langle q\rangle q_{i}-\frac{1}{2} \varphi\langle q\rangle^{2}
\end{aligned}
$$

Here the external field described by parameter $d$ is introduced. On the basis of eigenfunctions of harmonic oscillator the operators $p$ and $q$ have the form of the following infinite-dimensional matrices:

$q_{k j}=\sqrt{\frac{\hbar}{2 M \omega_{0}}}\left(\delta_{(k+1) j} \sqrt{k}+\delta_{k(j+1)} \sqrt{j}\right), p_{k j}=i \sqrt{\frac{M \hbar \omega_{0}}{2}}\left(\delta_{(k+1) j} \sqrt{k}-\delta_{k(j+1)} \sqrt{j}\right)$.

After substituting $q$ and $p$ in (6) according to (7), the Hamiltonian matrix has the following form (all terms are divided by $\hbar \omega_{0}$ ):

where

$$
\frac{\hat{H}}{\hbar \omega_{0}}=\hat{\alpha}-C_{1} \hat{\beta}+C_{2} \hat{\gamma}+C_{3} \hat{\sigma}(d+\varphi\langle q\rangle)-C_{4}\langle q\rangle^{2},
$$

$$
\begin{aligned}
C_{1}=\frac{\beta}{3}\left(\frac{\hbar}{2 M \omega_{0}}\right)^{\frac{3}{2}} \frac{1}{\hbar \omega_{0}}, & C_{2}=\frac{\gamma}{4}\left(\frac{\hbar}{2 M \omega_{0}}\right)^{2} \frac{1}{\hbar \omega_{0}}, \\
C_{3}=\left(\frac{\hbar}{2 M \omega_{0}}\right)^{\frac{1}{2}} \frac{1}{\hbar \omega_{0}}, & C_{4}=\frac{\varphi}{2} \frac{1}{\hbar \omega_{0}}
\end{aligned}
$$

and

$$
\begin{aligned}
\alpha_{i j}= & \delta_{i j} \frac{2 i-1}{2} \\
\beta_{i j}= & \delta_{i(j+1)} 3 j \sqrt{j}+\delta_{j(i+1)} 3 i \sqrt{i}+\delta_{i(j+3)} \sqrt{j(j+1)(j+2)}+ \\
& \delta_{j(i+3)} \sqrt{i(i+1)(i+2)}, \\
\gamma_{i j}= & \delta_{i j} 3\left(i^{2}+(i-1)^{2}\right)+\delta_{i(j+2)} 2(2 j+1) \sqrt{j(j+1)}+ \\
& \delta_{j(i+2)} 2(2 i+1) \sqrt{i(i+1)}+\delta_{i(j+4)} \sqrt{j(j+1)(j+2)(j+3)}+ \\
& \delta_{j(i+4)} \sqrt{i(i+1)(i+2)(i+3)}, \\
\sigma_{i j}= & \delta_{(i+1) j} \sqrt{i}+\delta_{i(j+1)} \sqrt{j} .
\end{aligned}
$$

For the purpose of the numerical treatment of the Hamiltonian matrix (8) we limit the dimension of this matrix to the required size. As the calculation of the dependencies of thermodynamical functions on the model parameters shows, the limitation of the size of Hamiltonian matrix (this corresponds to allowing for the finite number of harmonic oscillator levels) to $N=25$ is sufficient when $\frac{k T}{\hbar \omega_{0}} \leqslant 5$. All calculations in Section 2 are made within this approximation. 


\subsection{Order parameter and free energy}

For calculation of the mean value of coordinate $q_{i}$ which has a meaning of the order parameter we use the expression

$$
\langle q\rangle=\frac{\mathrm{Sp}\left(q e^{-\beta H}\right)}{\operatorname{Sp} e^{-\beta H}} .
$$

After the unitary transformation is made

$$
H_{d}=V^{-1} H V
$$

which diagonalizes the Hamiltonian matrix (8), we get

$$
\langle q\rangle=\frac{\operatorname{Sp}\left(\tilde{q} e^{-\beta H_{d}}\right)}{\operatorname{Sp} e^{-\beta H_{d}}}, \tilde{q}=V^{-1} q V .
$$

Note that $\langle q\rangle$ is contained in the Hamiltonian (8). Denoting $f=d+\varphi\langle q\rangle$, we derive the self-consistent system of equations:

$$
\left\{\begin{aligned}
\langle q\rangle & =\langle q\rangle(f, T) \\
f & =d+\varphi\langle q\rangle
\end{aligned}\right.
$$

Numerical solution of this system allows to obtain the dependence $\langle q\rangle=$ $\langle q\rangle(d)$ (figure 1a) for various values of $\frac{k T}{h \omega_{0}}$ (in all calculations here, $C_{1}=0.157$, $C_{2}=0.025$, and $C_{3} \varphi=-20$; these values of parameters correspond to the ones used in [5]). Substituting this function in the Hamiltonian (8), we can calculate the dependence of the free energy on the external field, according to the expression below:

$$
F(d, T)=-T \ln \operatorname{Sp} e^{-\beta H_{d}}-\frac{1}{2} \varphi\langle q\rangle^{2} .
$$

Figure 1 b represents the obtained result for $F=F(d)$.
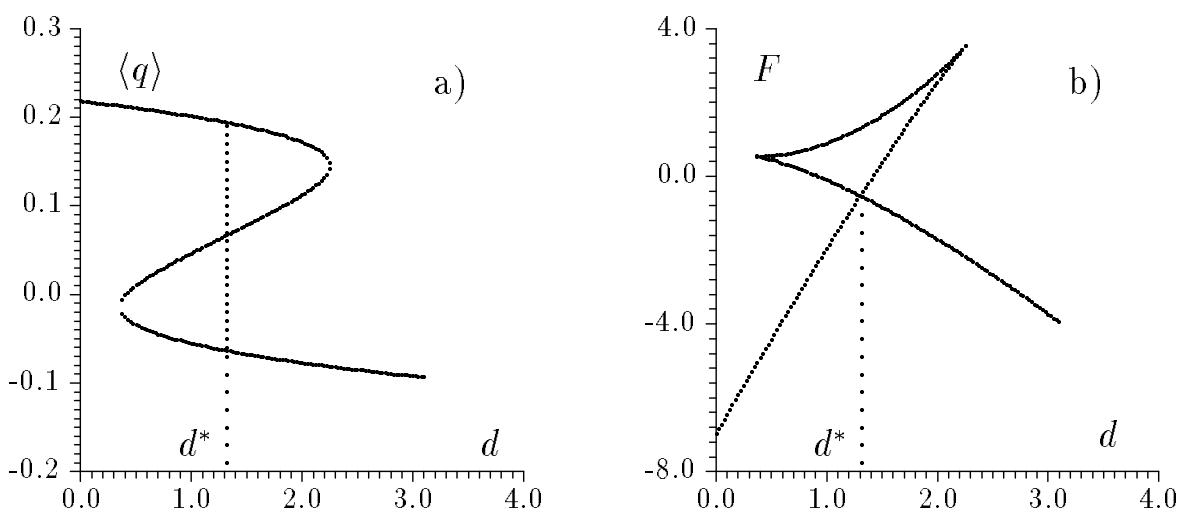

Figure 1. Dependencies of the order parameter and free energy on the external field, $\frac{k T}{\hbar \omega_{0}}=0.17$.

The dependencies shown in figure 1 are typical for the first order phase transition. The abscissa of the self-crossing point of the curve $F$ corresponds to the value $d^{*}$ at which the phase transition occurs, causing a jump-like change of the order parameter on figure 1a. Raising the temperature leads 
to the vanishing of the hysteresis-type of this dependence, i.e., to the disappearing of the phase transition.

The temperature increase also results in the decrease of $d^{*}$. Finding numerically $d^{*}$ for each value of the temperature and different values of $C_{1}$, we construct the phase diagrams $\left(d^{*}, T\right)$ which are shown in figure 2 .
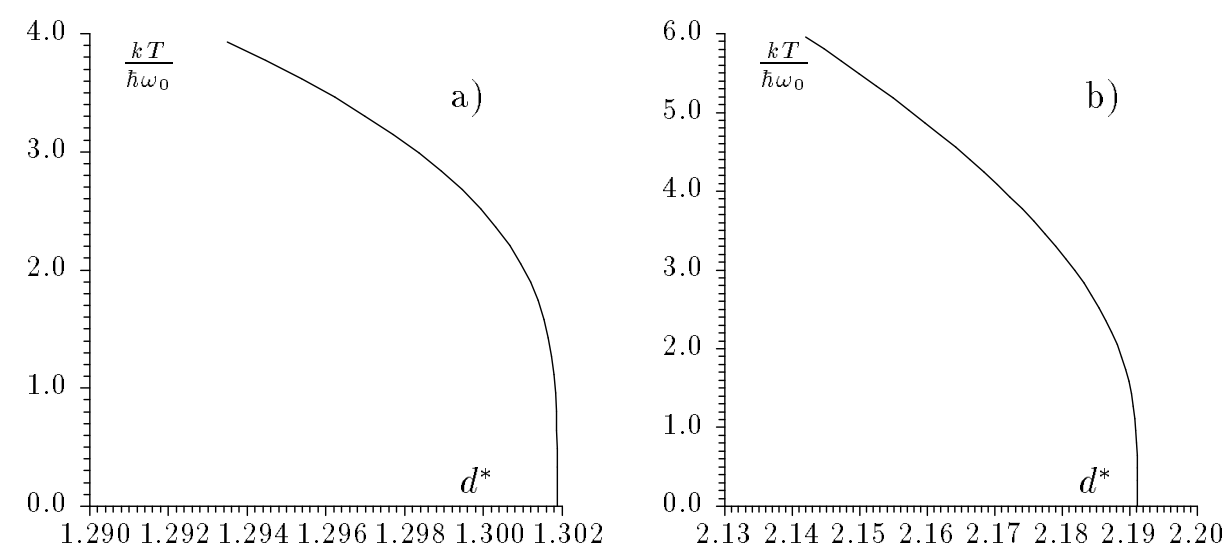

Figure 2. Phase diagram for different values of cubic anharmonicity: $C_{1}=0.157(\mathrm{a})$ and $C_{1}=0.197(\mathrm{~b})$.

The left end of the phase coexistence curve corresponds to the values of $d^{*}$ and $\frac{k T}{\hbar \omega_{0}}$ at which the phase transition disappears, having a meaning of the critical point $\left(\frac{k T}{\hbar \omega_{0}} \simeq 3.9, d_{c}^{*}=1.293\right.$ at $C_{1}=0.157 ; \frac{k T}{\hbar \omega_{0}} \simeq 6.0$, $d_{c}^{*}=2.143$ at $\left.C_{1}=0.197\right)$. It may be seen that the decreasing of the cubic anharmonicity leads to the lower value of the critical temperature. If $C_{1}=0$, the phase transition in the accepted approximation remains having a pure displacive nature. Thus, the peculiar feature of this model consists in the significant enhancement of the critical temperature due to the presence of the anharmonicity of third order. Figure 3 illustrates the dependence of the critical temperature on the value of cubic anharmonicity.

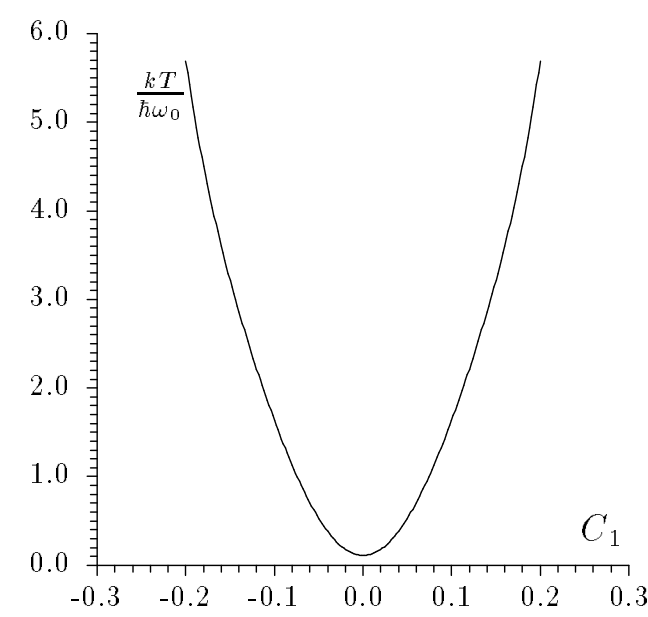

Figure 3. Dependence of the critical temperature on the value of cubic anharmonicity

On the basis of the obtained dependence $\langle q\rangle=\langle q\rangle(d)$, one can calculate 
the dielectric susceptibility function $\chi \sim-\frac{\partial\langle q\rangle}{\partial d}$. The dependencies $\chi^{-1}=$ $\chi^{-1}(d)$ are shown on figure $4 \mathrm{a}-4 \mathrm{~b}$. While the value of $\chi\left(\right.$ or $\left.\chi^{-1}\right)$ is finite at $\frac{k T}{\hbar \omega_{0}}=1.4$ (figure $4 \mathrm{a}$ ), which corresponds to the moving across the phase coexistence curve in the middle part of it (see diagram on figure $2 \mathrm{a}$ ), it tends to infinity at $\frac{k T}{\hbar \omega_{0}} \simeq 3.9$ (correspondingly, $\chi^{-1}$ reaches zero), i.e. as it approaches the critical point at the phase diagram (figure $4 \mathrm{~b}$ ).
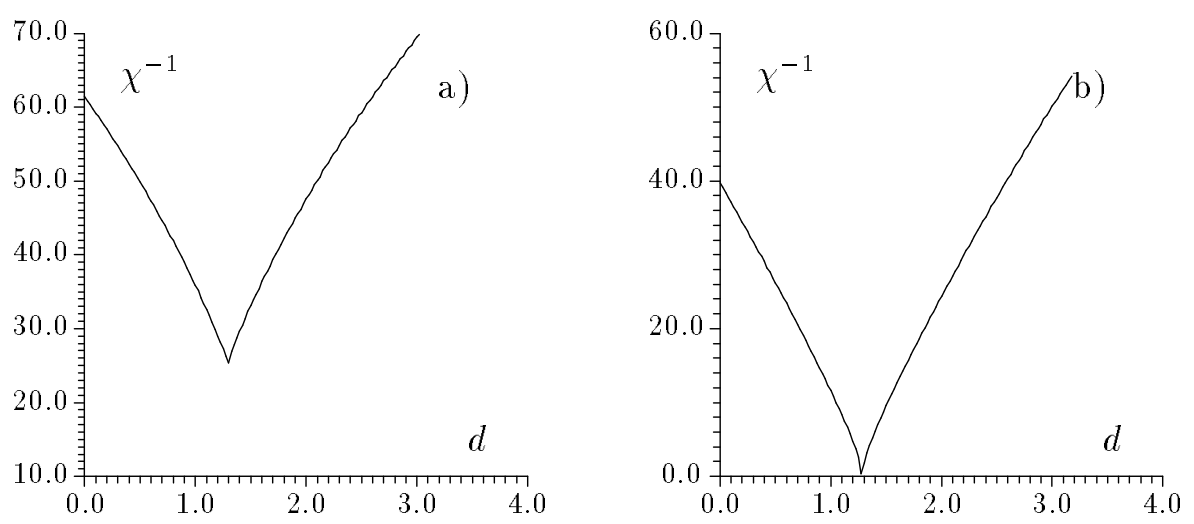

Figure 4. Inverse dielectric susceptibility as function of the external field, for the values of $\frac{k T}{\hbar \omega_{0}}=1.4$ and $\frac{k T}{\hbar \omega_{0}}=3.9$ which correspond to the middle part and to the left end of the phase coexistence curve shown in figure $2 \mathrm{a}$, respectively.

\subsection{Dependence of the order parameter and free energy on the temperature}

Solving numerically system (14) for certain values of $d$ we derive the dependence $\langle q\rangle=\langle q\rangle\left(\frac{k T}{\hbar \omega_{0}}\right)$. Using this dependence in the Hamiltonian (8) and diagonalizing it we obtain the free energy as a function of the temperature, according to (15). The obtained dependencies of the order parameter and free energy are shown in Figs. $5-7$ for $C_{1}=0.157$ and three values of $d$ : $d=1.4, d=1.3$, and $d=1.2$, which fall, respectively, to the right of, in the range of, and to the left of the phase coexistence curve (figure $2 \mathrm{a}$ ). The value of $d \simeq 1.293$ corresponds to the critical point; the dependence of the order parameter on the temperature for this value of $d$ is shown in figure 8 . 

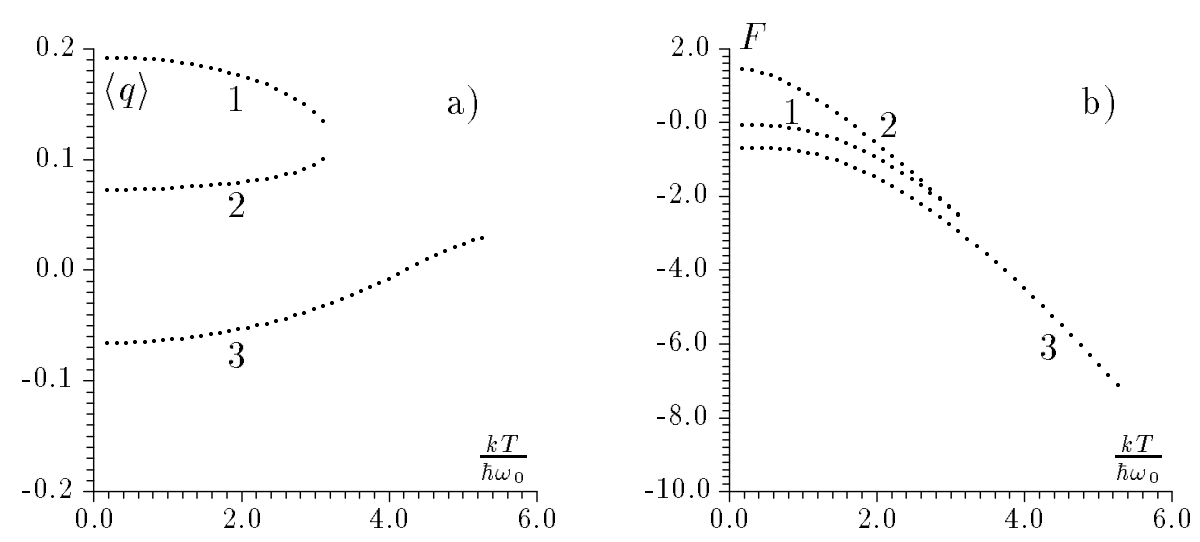

Figure 5. Plots of the order parameter and the free energy as functions of the $\frac{k T}{\hbar \omega_{0}}$ at $d=1.4, C_{1}=0.157$.
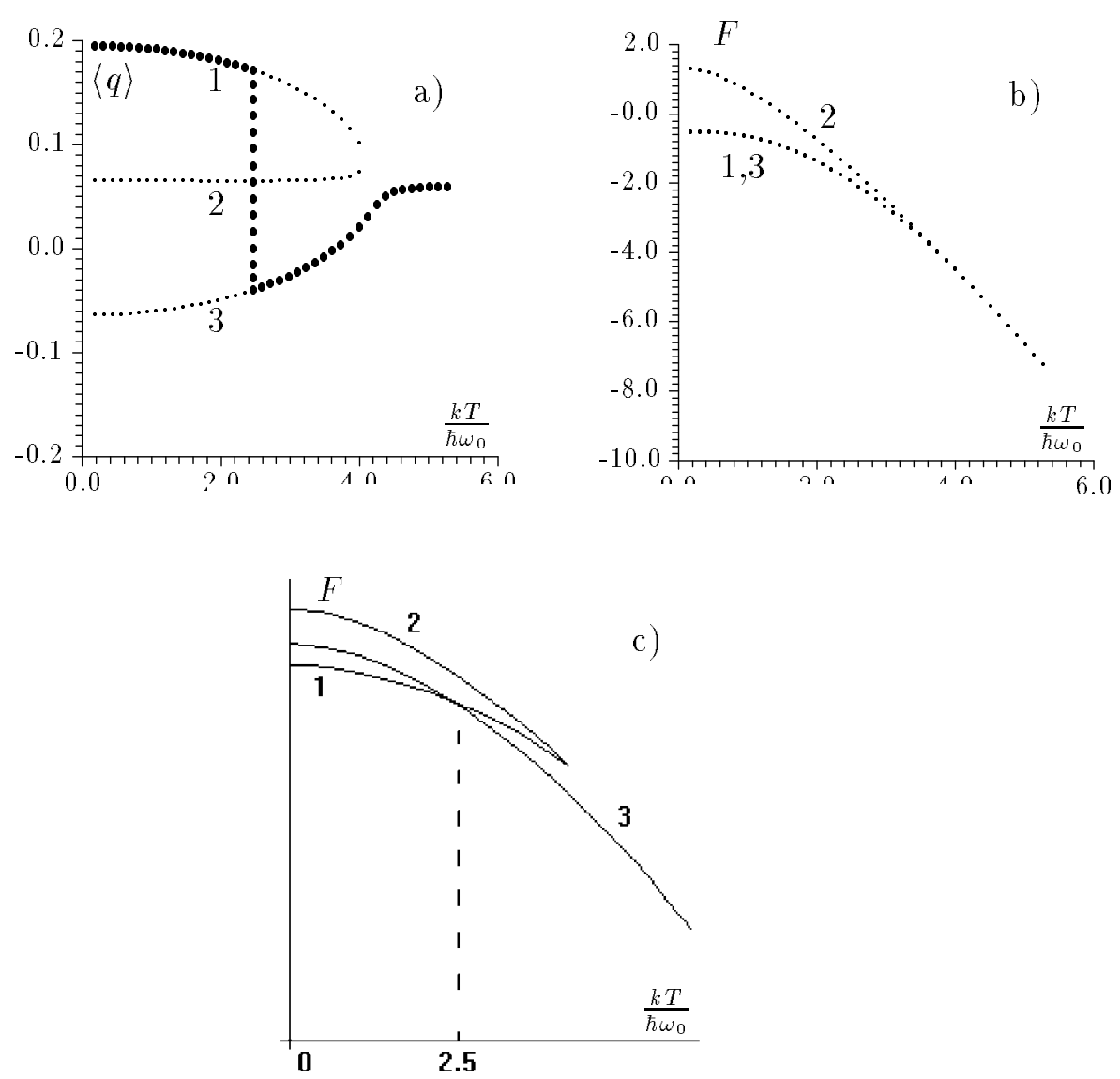

Figure 6. Plots of the order parameter and the free energy as functions of $\frac{k T}{\hbar \omega_{0}}$ at $d=1.3, C_{1}=0.157$. Since branches 1 and 3 on figure b) are very close to each other and can not be visually resolved, in figure c) the schematic picture of this dependence is given. 

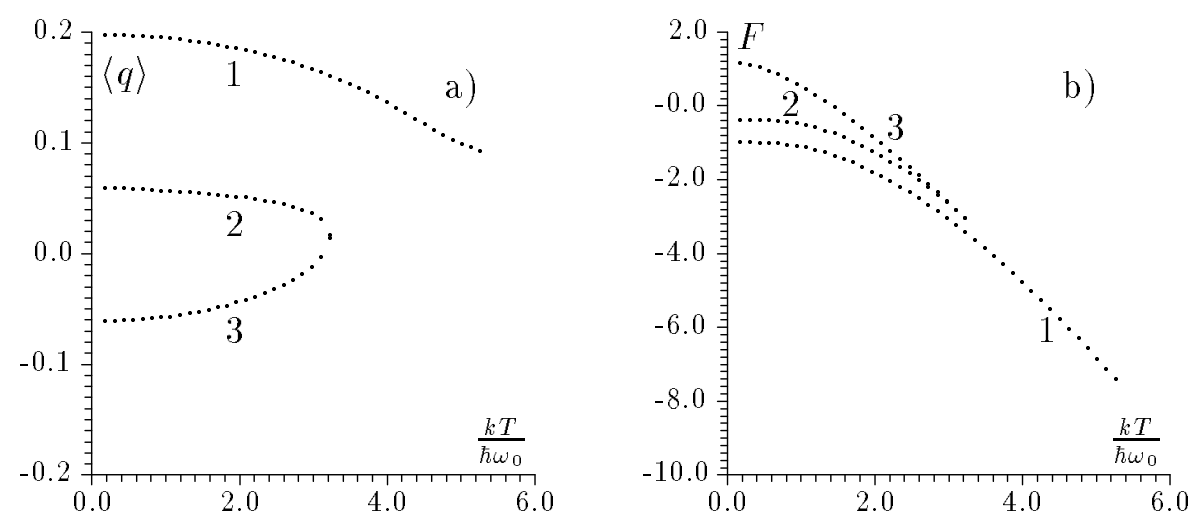

Figure 7. Plots of the order parameter and the free energy as functions of $\frac{k T}{\hbar \omega_{0}}$ at $d=1.2, C_{1}=0.157$.

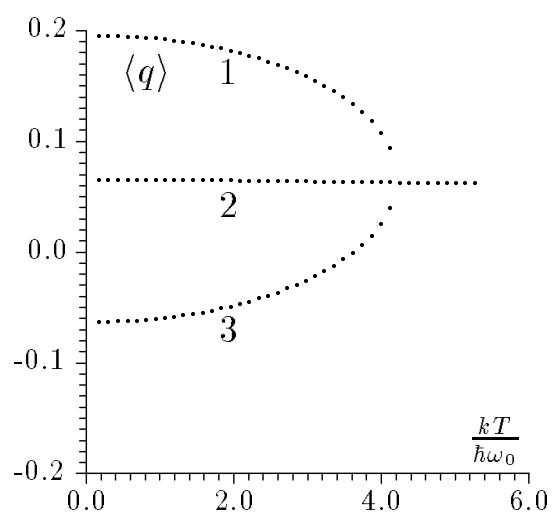

Figure 8. Plot of the order parameter as function of $\frac{k T}{\hbar w_{0}}$ at the critical point of the phase diagram in figure $2 \mathrm{a}, d=$ 1.293 .

The analysis of the free energy dependence on the temperature in the case of $d>1.3$ (figure 5), i.e., to the right side of the phase diagram curve in figure $2 \mathrm{a}$, shows that branch 3 of the dependence $\langle q\rangle=\langle q\rangle\left(\frac{k T}{\hbar \omega_{0}}\right)$ corresponds to the minimal value of the free energy, which means that the order parameter varies smoothly along branch 3 in this range of values of $d$. In the range $1.293<d<1.302$, where the first order phase transition occurs as the temperature varies (on moving across the phase coexistence curve), the minimal free energy corresponds to the jump of the order parameter from branch 3 to branch 1 (figure 6 ). At $C_{1}=0.157$ this takes place at the value $\frac{k T}{\hbar \omega_{0}}=2.5$, which corresponds to the first order phase transition (this transition may be interpreted as a point of lattice bistability [9]). When $d<1.293$ (the left part of the phase diagram), the order parameter varies smoothly along branch 1 in figure 7 ; no phase transition occurs in this case.

There is an another way which allows to determine the range of values 

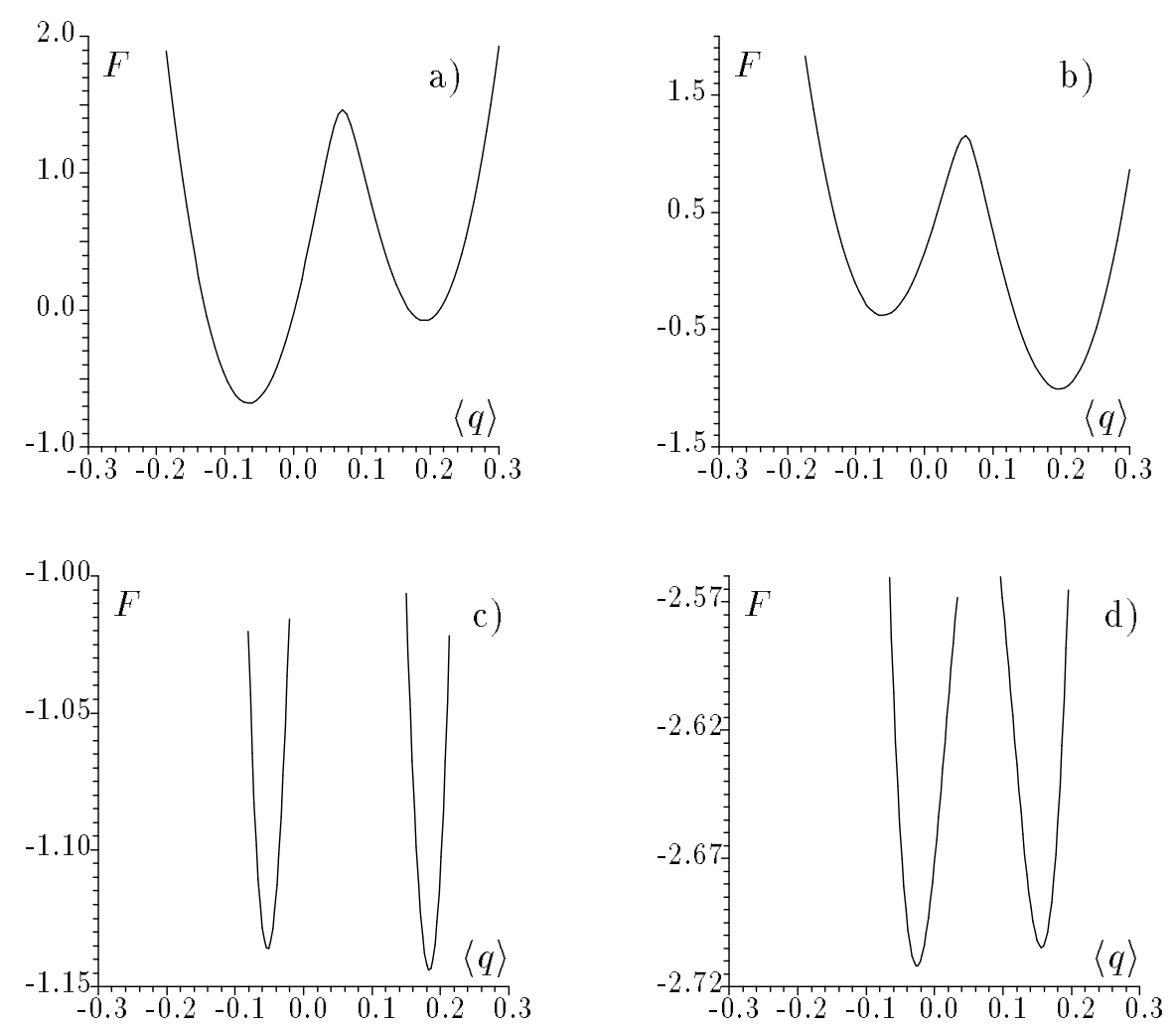

Figure 9. Dependencies of the free energy on the order parameter: $d=1.4(\mathrm{a}), d=1.2(\mathrm{~b}),\left(\frac{k T}{\hbar \omega_{0}}=0.2\right)$, and $d=1.3$, $\frac{k T}{\hbar \omega_{0}}=2(\mathrm{c}), \frac{k T}{\hbar \omega_{0}}=3(\mathrm{~d}), C_{1}=0.157$.

of $d$ where the phase transition occurs. We can calculate the dependence of the free energy on the order parameter, according to (15), for the fixed values of $\frac{k T}{\hbar \omega_{0}}$, and different values of $d$, which fall, respectively, to the right of, to the left of, and in the range of the phase coexistence curve.

We plot the dependencies of the free energy on the order parameter for $d=1.4$ and $d=1.2$ and $\frac{k T}{\hbar \omega_{0}}=0.2$ in Figs. $9 \mathrm{a}-9 \mathrm{~b}$. Comparing the dependence in figure $9 \mathrm{a}$ and the dependence of the order parameter on the temperature shown in figure 5a (case $d=1.4$ ) one concludes that the lowest minimal value of the free energy (at $\langle q\rangle \simeq-0.07$ ) corresponds to branch 3 of the figure $5 \mathrm{a}$. The same is true for all values of $\frac{k T}{\hbar \omega_{0}}$, therefore, the order parameter varies smoothly along this branch. The same smooth behaviour of the order parameter in the case of $d=1.2$ can be concluded by comparing figure $9 \mathrm{~b}$ and figure $7 \mathrm{a}$; in this case the lowest minimal value of the free energy in the figure $9 \mathrm{~b}($ at $\langle q\rangle \simeq 0.2)$ corresponds to the order parameter varying along branch 1 of the figure $7 \mathrm{a}$.

The case of $d=1.3$ for two values of $\frac{k T}{\hbar \omega_{0}}=2$ and $\frac{k T}{\hbar \omega_{0}}=3$ is represented on Figs. 9c - 9d. At $\frac{k T}{\hbar \omega_{0}}=2$ the minimal value of the free energy in figure $9 \mathrm{c}$ corresponds to the value of the order parameter $\langle q\rangle \simeq 0.18$, or branch 1 of the figure $6 \mathrm{a}$. However, at $\frac{k T}{\hbar \omega_{0}}=3$ the minimal value of the free energy in figure $9 \mathrm{~d}$ corresponds to the value of $\langle q\rangle \simeq-0.02$, or branch 3 of the 
figure 6 a. Between these two cases, at $\frac{k T}{\hbar \omega_{0}}=2.5$, a jump-like transition of the order parameter from branch 1 to branch 3 occurs (figure $6 \mathrm{a}$ ). This confirms the conclusion resulting from the analysis of the dependencies of the order parameter and free energy on the temperature.

We note that in the analysis presented above we did not raise the issue of how the possibility of the occurrence of the phase transition depends on the constant of the long-range interaction $\varphi$. One can see that as well as in the case of pure quadric model $\left(C_{1}=0\right)$, the phase transition disappears if $\varphi$ is less than the certain critical value $\left(|\varphi|<\left|\varphi^{*}\right|\right)$. The calculation of the wider class of the phase diagrams, taking into account the varying of $\varphi$, should be the subject of a separate investigation.

\section{Model with the Gaussian barrier-type potential}

\subsection{Model Hamiltonian and spectra}

While in the previous sections the potential considered possessed non-symmetrical form due to the presence of the cubic anharmonicity, we consider now the potential of the symmetrical form (4):

$$
V_{G}\left(q_{i}\right)=\frac{M \omega_{0}^{2} q_{i}^{2}}{2}+C e^{-B q_{i}^{2}}
$$

We use the same approach as we did in the previous sections, that is we construct the Hamiltonian matrix $H_{m n}$ on the basis of the normalized eigenfunctions of the harmonic oscillator (with the potential energy given by the first term in (16)), using relations (1) and (5), with $V_{G}\left(q_{i}\right)$ in the form above. Then

$$
\frac{H_{m n}}{C}=\delta_{m n}\left(n+\frac{1}{2}\right) \gamma \frac{2 \hbar}{M \omega_{0}}+V_{m n}+\frac{(d+\varphi\langle q\rangle)\langle q\rangle q_{m n}}{C},
$$

where

$$
\begin{gathered}
\gamma=\frac{M \omega_{0}^{2}}{2 C}, \\
V_{m n}=\frac{1}{\sqrt{\pi}} \frac{1}{\sqrt{2^{n+m} n ! m !}} \int_{-\infty}^{\infty} e^{-\left(1+B \frac{\hbar}{M \omega_{0}}\right) x^{2}} H_{n}(x) H_{m}(x) \mathrm{d} x .
\end{gathered}
$$

Here $H_{n}(x)$ are the Hermite polynomials, and $q_{m n}$ are defined in (7). Below are the relations used for calculation of the integral appearing in (19):

$$
\begin{gathered}
\int_{-\infty}^{\infty} e^{-p x^{2}}\left\{\begin{array}{l}
H_{2 m+1}(x) H_{2 n+1}(x) \\
H_{2 m}(x) H_{2 n}(x)
\end{array}\right\} \mathrm{d} x=(2 m+\delta) !(2 n+\delta) ! \sqrt{\frac{\pi}{p}} \times \\
\times \sum_{k=0}^{m i n(m, n)} \frac{1}{(m-k) !(n-k) !(2 k+\delta) !}\left(\frac{1-p}{p}\right)^{m+n-2 k}\left(\frac{2}{p}\right)^{2 k+\delta}, \\
\delta=\left\{\begin{array}{l}
1 \\
0
\end{array}\right\} ;
\end{gathered}
$$




$$
\int_{-\infty}^{\infty} e^{-p x^{2}} H_{2 m}(x) H_{2 n+1}(x) \mathrm{d} x=0 .
$$

Using relations (16) and (18), we write

$$
\frac{V_{G}(q)}{C}=\gamma q^{2}+e^{-B q^{2}} .
$$

The condition of $\frac{V_{G}(q)}{C}$ to have two minima is $\gamma<B$. Assuming in the calculations below $\stackrel{C}{B}=1$, this condition rewrites as $\gamma<1$. Then the meaning of $\gamma$ is that the closer is the value of $\gamma$ to 1 the smaller is the height of the Gaussian barrier (if $\gamma=1$, the potential (4) has only one minimum the barrier disappears).

Transforming the matrix $H$ to the diagonal form in the absence of the external field $d$, we derive the spectra for the various values of $\gamma$. Approximately 35 lowest levels of the spectra are plotted on figure 10 at $\gamma=0.4$ and $\gamma=0.7 ; 32$ energy values $\frac{\epsilon}{\hbar \omega_{0}}$ at $\gamma=0.4$ and $\gamma=0.7$ are also given in tables 1 and 2, respectively. (We note that within the range of the model parameters considered, particularly when $\gamma=0.4-0.7$, the number of harmonic oscillator levels which were needed to be taken into account to provide the required accuracy $\left(\frac{\Delta \varepsilon}{\varepsilon}=7 * 10^{-5}\right)$ of calculations of this part of spectrum or the required size of the Hamiltonian matrix was $N \simeq 100$ ).

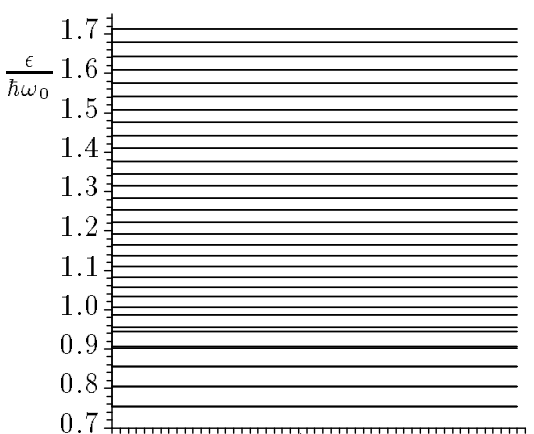

a)

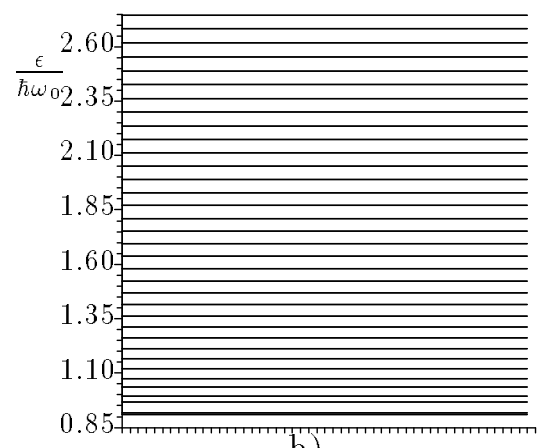

b)

Figure 10. Spectra $\frac{\epsilon}{\hbar \omega_{0}}$ at $\gamma=0.4$ (a) and $\gamma=0.7$ (b).

Table 1. Eigenvalues $\frac{\epsilon}{\hbar \omega_{0}}$ at $\gamma=0.4$.

\begin{tabular}{|c|c|c|c|c|c|c|c|}
\hline 0.75315 & 0.8548 & 0.9446 & 1.0325 & 1.1364 & 1.2525 & 1.3772 & 1.5081 \\
\hline 0.75317 & 0.8563 & 0.9559 & 1.0559 & 1.1644 & 1.2830 & 1.4094 & 1.5416 \\
\hline 0.8052 & 0.9014 & 0.9869 & 1.0826 & 1.1932 & 1.3140 & 1.4419 & 1.5754 \\
\hline 0.8055 & 0.9061 & 1.0052 & 1.1088 & 1.2226 & 1.3454 & 1.4748 & 1.6094 \\
\hline
\end{tabular}

Approximately ten lowest levels of the spectrum on figure 10a $(\gamma=0.4)$ are doublets (which is a well-known consequence of the presence of doublewell potential), although the first three doublets can not be visually resolved 
Table 2. Eigenvalues $\frac{\epsilon}{\hbar \omega_{0}}$ at $\gamma=0.7$.

\begin{tabular}{|l|l|l|l|l|l|l|l|}
\hline 0.908 & 1.037 & 1.212 & 1.415 & 1.636 & 1.870 & 2.113 & 2.362 \\
\hline 0.917 & 1.076 & 1.260 & 1.469 & 1.694 & 1.930 & 2.174 & 2.426 \\
\hline 0.967 & 1.120 & 1.311 & 1.524 & 1.752 & 1.990 & 2.237 & 2.489 \\
\hline 0.993 & 1.165 & 1.362 & 1.579 & 1.810 & 2.051 & 2.299 & 2.553 \\
\hline
\end{tabular}

(for example, the width of the first doublet $\Delta \varepsilon_{1}$ is much smaller than the distance between the first and the second doublet $\varepsilon_{12}: \frac{\Delta \varepsilon_{1}}{\varepsilon_{12}} \simeq 4 * 10^{-4}$, see table 1). As one moves to the middle and upper parts of the spectrum, one can see that the spectrum gradually transforms into the system of equally distanced energy levels which is typical for the harmonic oscillator, thus proving that the potential in form (4) is the low-temperature anharmonicity potential indeed. If we decrease the height of the Gaussian barrier (figure $10 \mathrm{~b}, \gamma=0.7)$, the number of doublets in the spectrum decreases. The crossover between doublet levels and nearly equally distanced parts of the spectrum takes place in this case at the closer to ground state values of energy. Finally, if $\gamma>1$, or if the potential (20) has only one minimum, all spectrum becomes more and more closer to the system of equally distanced energy levels.

\subsection{Order parameter and free energy. Phase diagram}

Applying the procedure described in Section 2.2 (relations (11-15)) to the Hamiltonian (17), we derive the dependencies of the order parameter and free energy on the external field which are shown on figure 11. Unlikely to the case of non-symmetrical potential (3), where the value $d=d^{*}$ at which the jump-like change of the order parameter occurs was different from zero (see figure 1 and figure 2), the phase transition takes place now at $d^{*}=0$. This is the result of the symmetrical form of potential (4). The temperature change does not influence the value of $d^{*}$. Therefore, the phase coexistence curve shown on figure 12 is the vertical line. Its end's position (the critical point) depends on the value of $\gamma$. Figure 13 illustrates the connection between the critical temperature $\frac{k T}{\hbar \omega_{0}}$ and the values of parameter $\gamma$.

As it follows from figure 13 , for the smaller value of $\gamma$, i.e., the larger height of the Gaussian barrier, the larger value of the critical temperature $\frac{k T}{\hbar \omega_{0}}$ is needed to destroy the hysteresis-type dependence of the order parameter on $d$, or to destroy the phase transition. We note that even when $\gamma>1$, i.e. when the potential (4) transforms from two-minima to the oneminimum potential, the system still undergoes a phase transition (which in this case is displacive in character, while it is of order-disorder type in case of $\gamma<1$, when the potential has two minima): as it follows from figure 13, the phase transition in the system occurs until $\gamma \simeq 4$.

\subsection{Dependence of the order parameter and free energy on the temperature}

The dependencies of the order parameter and the free energy on the temperature are derived by the calculations similar to those in Section 2.3. We 

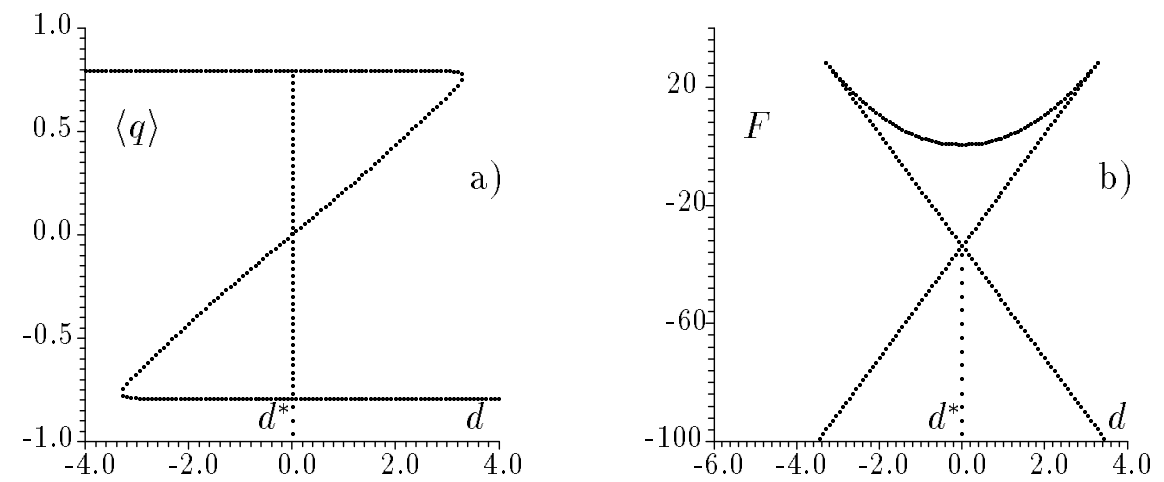

Figure 11. Dependence of the order parameter and free energy on the external field, $\frac{k T}{\hbar \omega_{0}}=0.2, \gamma=0.4$.

plot dependencies of $\langle q\rangle=\langle q\rangle\left(\frac{k T}{h \omega_{0}}\right)$ and $F=F\left(\frac{k T}{h \omega_{0}}\right)$ for $d=0.1$ and $d=0$ (see Figs. $14-15$ ).

In the range $d>0$ (figure 14) branch (3) of $\langle q\rangle=\langle q\rangle\left(\frac{k T}{\hbar \omega_{0}}\right)$ corresponds to the minimal value of the free energy which therefore means that $\langle q\rangle$ varies smoothly along this branch. The same is true with interchanging of roles of 1 and 3 branches in the case of $d<0$. These two cases $(d \neq 0)$ are similar to those represented in Figs. 5 and 7.

The Case of $d=0$, which corresponds to the phase diagram of figure 12 , is presented on figure 15. Branches 1 and 3 of $\langle q\rangle=\langle q\rangle\left(\frac{k T}{\hbar \omega_{0}}\right)$ (figure 15a) correspond to one "degenerated" branch of $F=F\left(\frac{k T}{\hbar \omega_{0}}\right)$ (figure 15b) and therefore are equivalent in terms of the possible values of $\langle q\rangle$ (see figure $15 \mathrm{a})$. At the branching point $\left(\frac{k T}{\hbar \omega_{0}} \simeq 10.4\right)$ the appearance of the non-zero value of $\langle q\rangle$ corresponds to the second order phase transition.

We also plot the dependencies $F=F(\langle q\rangle)$ for $d=0.1$ and $d=0$ in figure 16. For $d=0.1$ the minimal value of $F$ corresponds to branch 3 of $\langle q\rangle$ on figure $14 \mathrm{a}(\langle q\rangle \simeq-0.54)$, thus showing that $\langle q\rangle$ varies along this branch; for $d=0 F=F(\langle q\rangle)$ has two equivalent minima at $\langle q\rangle \simeq \pm 0.54$, which correspond to branches 1 and 3 on figure $15 \mathrm{a}$. This is in agreement with the above given conclusion and confirms that in this approximation the phase transition at the temperature change is of the second order. The self-consistent phonons approximation results, however, in the phase transition of the first order [11]. The reason for such a discrepancy is the different character of both approaches used: the first approach does not fully takes into account the long-range interaction, and the second one treats approximately the local anharmonicity. More consistent description of the phase transition in the framework of the proposed in this paper scheme certainly requires the consideration of quantum fluctuations relatively to the mean field.

\section{Conclusions}

The approach based on the numerical solution of the single ion problem in the crystal lattice is developed for the investigation of the local anhar- 


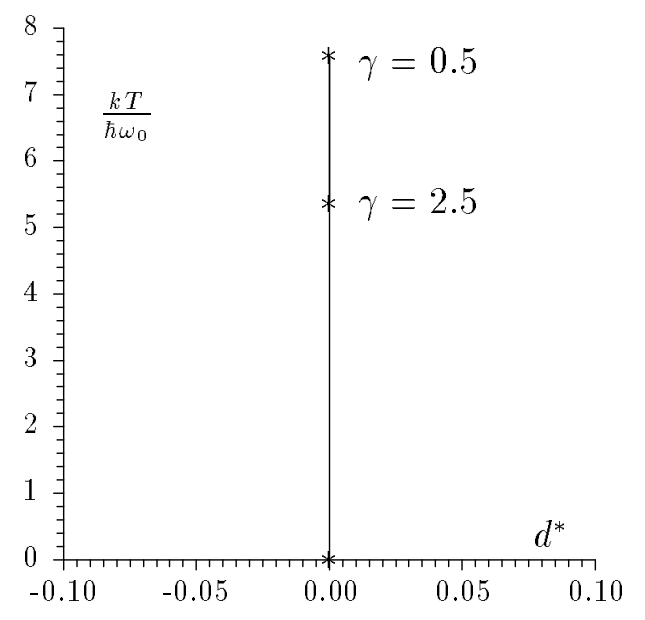

Figure 12. Phase diagram for two different values of $\gamma: \gamma=0.5$ and $\gamma=2.5$.

monicity model of structural phase transitions in solids for the more general non-symmetrical form of the potential (3) which effects on the ions, as well as the symmetrical potential of a special form (4). The long-range interactions are taken into account in the mean-field approximation. The dependencies of the dynamic and thermodynamic functions on the external field and the temperature are derived. In the case when the system is described by the potential (3), these dependencies testify that the system undergoes the first order phase transition, with the jump-like change of the order parameter $\langle q\rangle$, as the external field varies as well as the temperature. It is established that the increase of the cubic anharmonicity constant leads to the increase of the critical temperature. In the case when the system is described by the potential (4), it undergoes the first order phase transition as the external field varies and the second order phase transition as the temperature varies at zero value of the field. The increase of the Gaussian barrier height results in the increase of the critical temperature. The phase diagrams $(d, T)$ of the models described by (3) and (4) are built.

We would like to point out that the results obtained from the consideration of the problem with non-symmetrical potential (3) regarding the character of the phase transitions generally comply with the conclusions of [6] and [14], in which the similar investigations were related to the pseudospinelectron model of HTSC systems. Both in the those cases, and in the model (3), the potential asymmetry leads to the deviation of the phase coexistence curve from the vertical line on the plane (temperature-field) and to the appearance of the first order phase transitions (which may manifest themselves in the instability of the dielectric susceptibility [6]) as the temperature varies in the certain range of the parameter which defines mentioned asymmetry. Existence of this phase transition corresponds to the bistability of the system with the local potential (3) mentioned in [9]. However, the character of the dependence $\langle q\rangle=\langle q\rangle\left(\frac{k T}{\hbar \omega_{0}}\right)$ (shown on figure 6) differs from the one derived in [9] which is the result of using different approximations.

The substantial conclusion can be made from the investigation of the single-ion spectra made in Section 3: the existence of two parts of the spec- 


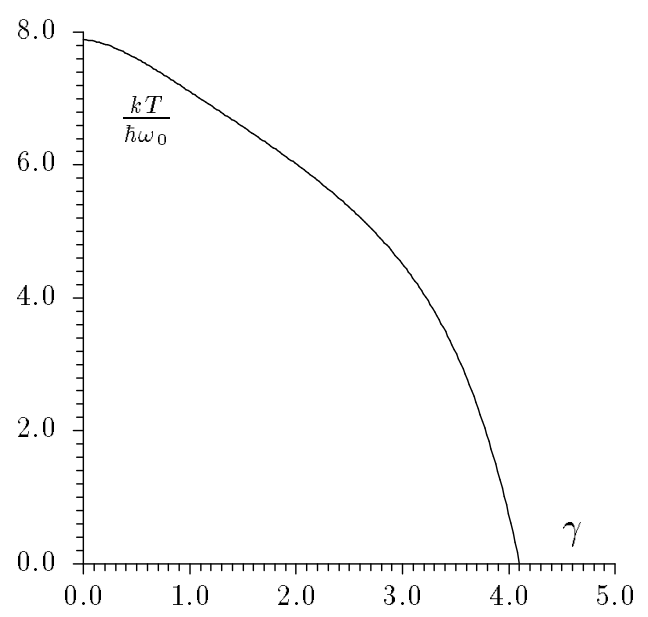

Figure 13. Dependence of the critical temperature on $\gamma$.
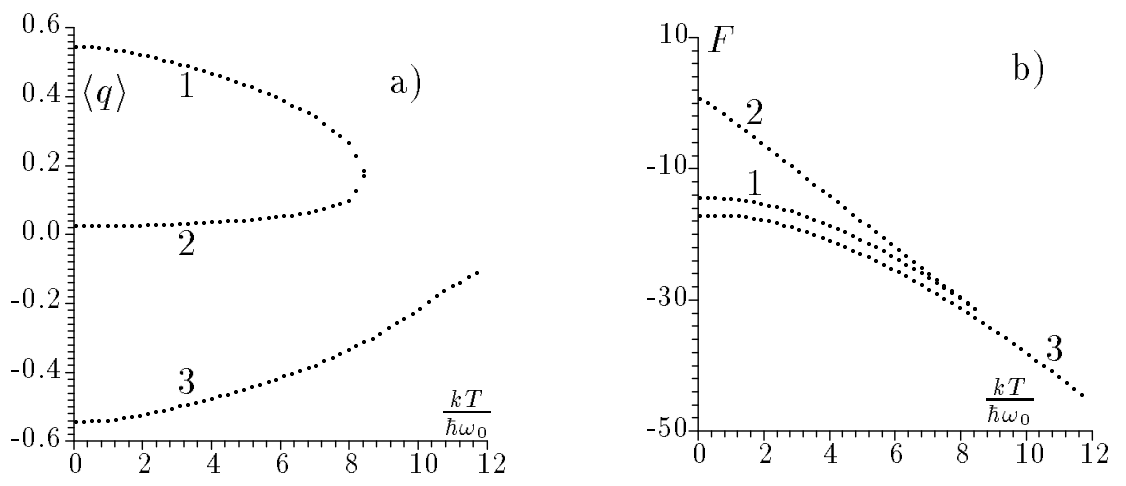

Figure 14. Plots of the order parameter and free energy as functions of $\frac{k T}{\hbar \omega_{0}}$ at $d=0.1, \gamma=0.4$.

trum of different character, with quasidoublet (at the lower values of energy) and quasi equally distanced (at the higher values of energy) structures. The crossover from one part to another is relatively sharp and takes place in the range of energies which are of the height of potential barrier by the order of magnitude (the possibility of such a transformation in the spectrum was foreseen in [15] and was formulated in terms of simple model). As it follows from the results obtained, the model (4) incorporates phase transitions of displacive $(1<\gamma<4)$, as well as order-disorder types $(\gamma<1)$.

The approach used in this paper may be applied to the investigation of the dynamics of the considered models and thermodynamics of the phase transitions using approximations higher than the mean field approximation. In doing it, the capabilities of this seminumerical approach can be demonstrated and its comparison with approximate analytical schemes can be made. 

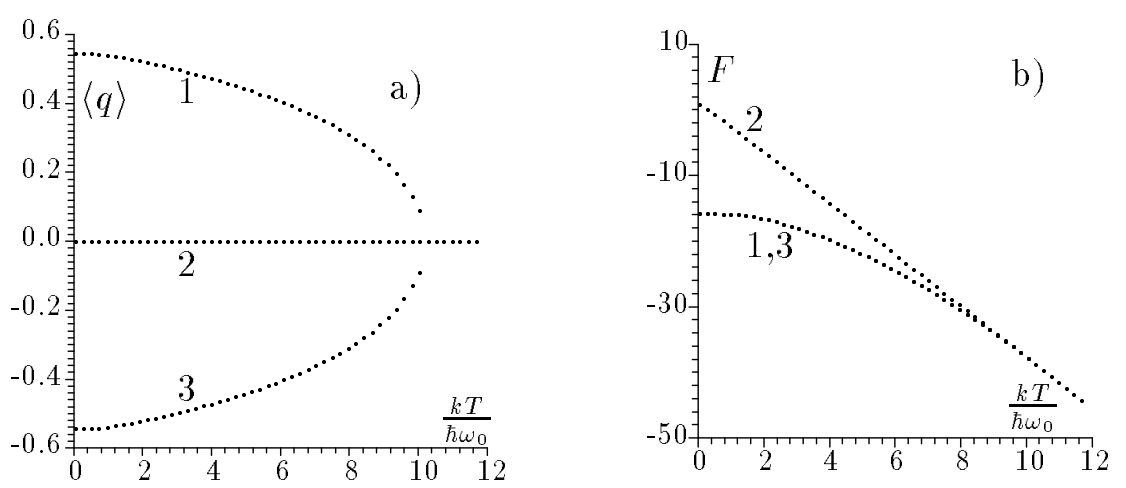

Figure 15. Plots of the order parameter and free energy as functions of $\frac{k T}{\hbar \omega_{0}}$ at $d=0, \gamma=0.4$.
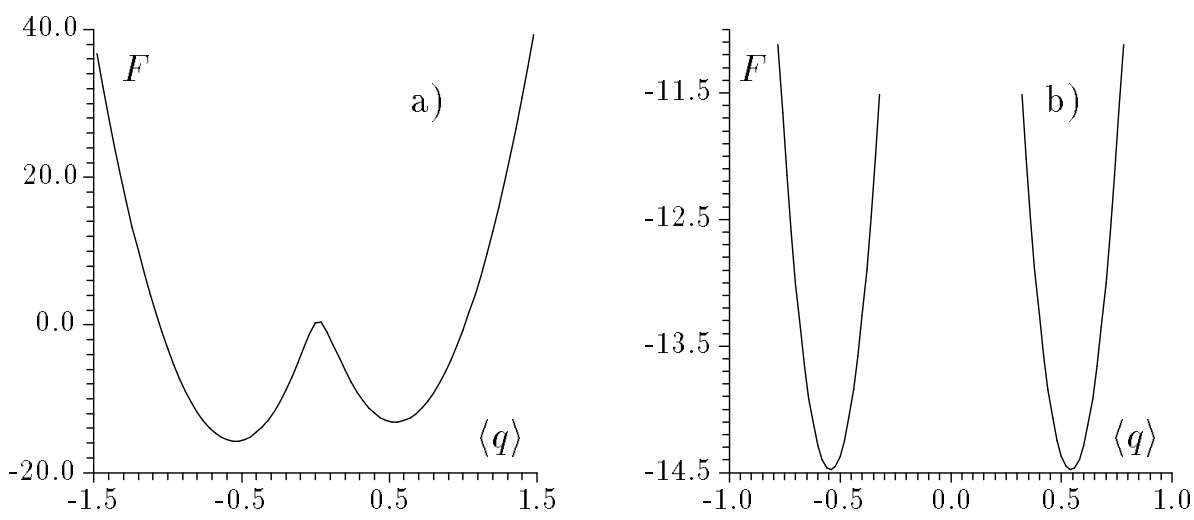

Figure 16. Dependence of the free energy on the order parameter: $d=0.1(\mathrm{a}), d=0$ (b), $\frac{k T}{\hbar \omega_{0}}=0.2 ; \gamma=0.4$.

\section{Acknowledgments}

This work was supported in part by the International Soros Science Education Program through grant No. SPU 062062. The authors would like to acknowledge O. D. Danyliv for the assistance in some of the calculations.

\section{References}

[1] Aksenov V.L., Plakida N.M., Stamenkovic S. Neutron scattering by ferroelectrics. // Moscow, Energoatomizdat, 1984, p. 55-71 (in Russian).

[2] Gillis N., Koehler T. Phase transitions in a simple model ferroelectric - comparison of exact and variational treatments of a molecular-field Hamiltonian. // Phys. Rev. B, 1973, vol. 9, No 9, p. 3806-3818.

[3] Bishop A., Martin R., Muller K., Tesanovic Z. Superconductivity in oxides: toward a unified picture. // Z. Phys. B, Condensed Matter, 1989, vol. 76, No 1 , p. 17-24.

[4] Muller K.A. // Phase transitions, 1988 (Special issue).

[5] Frick M., von der Linden W., Morgenstern I., Raedt H. Local anharmonic vibrations, strong correlations and superconductivity: A quantum simulation study. // Z. Phys. B, Condensed Matter, 1990, vol. 81, No 2, p. 327-335. 
[6] Stasyuk I.V., Shvaika A.M. A model with local anharmonicity in theory of HTSC systems: correlation functions and "transverse" dielectric susceptibility. // Cond. Matt. Phys., 1994, No 3, p. 134-175.

[7] Krantz M., Thomson C., Mattausch Hj., Cardona M. Raman-active phonons and mode softening in superconducting $\mathrm{HgBa}_{2} \mathrm{CuO}_{4}+_{\delta} . / /$ Phys. Rev. B, 1994, vol. 50, No 2, p. 1165-1170.

[8] Sayko A.P., Gusakov V.E. // On reason of forming of two superconductive phases in Yttrium-Barium Cuprates. // Journal of Experimental and Theoretical Physics, 1995, vol. 108, No 2(8), p. 757-764 (in Russian).

[9] Sayko A.P., Gusakov V.E., Kuzmin V. On the effect of the bistable behaviour of apex oxygen on superconducting transition in $\mathrm{YBa}_{2} \mathrm{Cu}_{3} \mathrm{O}_{7}-\delta$. // Journal of Experimental and Theoretical Physics Letters, 1992, vol. 56, No 8, p. 425428 (in Russian).

[10] Yukhnovskii I.R., Idzyk I.M., Kolomiets V.O. Investigation of a homogeneous many-particle system in the vicinity of the critical point. // J. Stat. Phys., 1995, vol. 80, No 1/2, p. 405-444.

[11] Plakida N. A ferroelectric model with strong low temperature anharmonicity. // Low Temperature Physics, 1981, vol. 7, No 5, p. 644-651 (in Russian).

[12] Grigas J., Kalesinskas V., Stasyuk I.V. Soft microwave mode in SbSJ-type crystals. // Ferroelectrics, 1984, vol. 55, No 1, p. 31-34.

[13] Coon J.B., Naugle N.W., McKenzie R.D. The investigation of doubleminimum potentials in molecules. // Journal of Molecular Spectroscopy, 1966, vol. 20 , p. $107-129$.

[14] Danyliv O.D., Stasyuk I.V. The analysis of ferroelectric type instabilities in the two-sublattice model of high temperature superconducting systems. // Cond. Matt. Phys., 1996, No 7, p. 163-177.

[15] Stasyuk I.V. Dynamics of simple model of ferroelectric phase transition. // Kyiv, 1975, 22 p. Preprint of the Academy of Sciences of USSR, ITF; ITF75-108R, (in Russian).

\title{
ДОСЛІДЖЕННЯ ЛОКАЛЬНО АНГАРМОНІЧНИХ МОДЕЛЕЙ СТРУКТУРНИХ ФАЗОВИХ ПЕРЕХОДІВ. НАПІВЧИСЛОВИЙ ПІДХІД
}

\author{
I.В.Стасюк, К.О.Траченко
}

В контексті опису структурних фазових переходів в твердих тілах ангармонічній потенціал, що діє на іон в кристалічній гратці, вибирається у загальній несиметричній формі, що на додаток до ангармонізму четвертого порядку містить ангармонізм третьої степені, а також у симетричній формі 3 гаусовим бар'єром. Застосовується підхід, що базуеться на чисельній діагоналізації матриці одноіонного гамільтоніану. Виявлено особливість енергетичного спектру системи з гаусовим бар'єром в одноіонному потенціалі, що полягає в існуванні областей з квазідублетною і квазіеквідистантною структурою, кросовер між якими відбувається у вузькому інтервалі енергій. Отримано залежності параметра порядку, вільної енергї і діелектричної сприйнятливості від зовнішнього поля і параметра порядку та вільної енергії від температури. Побудовано фазову діаграму (поле, температура) і досліджено вплив ангармонізму на криву рівноваги фаз і положення критичної точки. Показано, що асиметрія локального потенціалу приводить до відхилення кривої рівноваги фаз від вертикалі на площині (поле, температура) і появи фазового переходу першого роду при зміні температури. 